\title{
Reality of Space and Time
}

\author{
Tower Chen ${ }^{1}$, Zeon Chen ${ }^{2}$ \\ ${ }^{1}$ Retiree from Unit of Mathematical Sciences, College of Natural and Applied Sciences, University of Guam, UOG Station, \\ Mangilao, Guam, USA \\ ${ }^{2}$ Independent Researcher, Berkeley, CA, USA \\ Email: tower_c@yahoo.com,zeon_chen@yahoo.com
}

How to cite this paper: Chen, T. and Chen, Z. (2017) Reality of Space and Time. Journal of Applied Mathematics and Physics, 5, 774-784.

https://doi.org/10.4236/jamp.2017.54066

Received: December 24, 2016

Accepted: April 9, 2017

Published: April 12, 2017

\begin{abstract}
In Newton's classical physics, space and time are treated as absolute, independent quantities and can be discussed separately. In Special Relativity, Einstein proved that space and time are relative and dependent and therefore must not be treated separately. Minkowski adopted four-dimensional spacetime frames (4-d s-t frames), which indirectly revealed the dependency of space and time with the addition of a constraint for an event interval. We are not able to visualize 4-d s-t frames. Since space and time are inseparable, three-dimensional space-time frames (3-d s-t frames) can be constructed by embedding time into space to directly show the interdependency of space and time. Time contraction and length contraction can also be depicted graphically using 3-d s-t frames. We have much better understanding reality of space and time in 3-d s-t frames. This will lead to Contextual Reality for better understanding the universe.
\end{abstract}

\section{Keywords}

Special Relativity, Four-Dimensional Space-Time Frames, 4-D S-T Frames, Three-Dimensional Space-Time Frames, 3-D S-T Frames, Time Contraction, Length Contraction, Contextual Attributes, Contextual Reality

\section{Introduction}

In order to describe the position of a static object in space, Descartes constructed three axes $(x, y, z)$ perpendicular to one another and used the $(x, y, z)$ coordinate to represent the position of this static object along $x$-axis, $y$-axis, and $x$-axis. The coordinate system is called the Cartesian frame. The properties of algebraic functions can be depicted in the Cartesian frame. It led to calculus, which is foundation of analytical mathematics.

In order to describe the position of a moving object, Galileo constructed a time axis which is perpendicular to each of the three special axes, and used the 
$(x, t),(y, t)$, and $(z, t)$ coordinate frame to represent the position of this moving object along $x$-axis, $y$-axis, and $x$-axis. Coordinates of this kind are called Galileanframes. It led to the Galileo transformation, which is foundation of Newton's classical physics.

In order to describe the position of a moving object in Special Relativity, Minkowski constructed a time axis, $c t$, which is simultaneously perpendicular to the three special axes, where $c$ represents the speed of light In order to achieve this, he first treated space and time independently by representing them as separate $(x, t),(y, t)$, and $(z, t)$ coordinate frames, then made space and time dependent with the addition of the following constraint: $x^{2}+y^{2}+Z^{2}-(c t)^{2}=$ const. He use this $(c t, x, y, z)$ coordinate frame to represent the position of objects moving along $\mathrm{x}$-axis, $\mathrm{y}$-axis, and $\mathrm{z}$-axis. Coordinates of this kind are called Minkowski's four-dimensional space-time frames (4-d s-t frames), which cannot be visualized. In Special Relativity, Einstein derived the Lorentz transformation, time dilation, and length contraction, which can be drawn in 4-d s-t inertial frames, but the graph is very complicated [1].

The following are quotes from the book The Trouble with Physics authored by Lee Smolin in 2006. "More and more, I have the feeling that quantum theory and general relativity are both deeply wrong about the nature of time. Descartes and Galileo made a most wonderful discovery. In this way, time is represented as if it were another dimension of space. This spatialization of time is useful but may be challenged. We have to find a way to unfreeze time -- to represent time without turning it into space." We believe that the use of three-dimensional space-time frames (3-d s-t frames)is a potential means to address this issue regarding the representation of time.

In order to describe the position of a moving object in Special Relativity, we construct polar coordinates on the $x-y$ plane, $y-z$ plane, and $z-x$ plane to represent time. The unit for the radius of polar coordinate is light-sec or period $\mathrm{T}$, and the unit for $\mathrm{x}$-axis, $\mathrm{y}$-axis, and $\mathrm{z}$-axis in space is light-sec or wavelength $\lambda$. We use coordinates to represent the position of this moving object along $\mathrm{x}$-axis, $\mathrm{y}$-axis, and $\mathrm{z}$-axis in a frame. This process of embedding time into space shows their dependence [2]. This coordinate system with the form of (x-ct, $y$-ct, $\mathrm{z}$-ct), is called three-dimensional space-time frame (3-d s-t frame), which is depicted in Figure 1. In the proposed 3-d s-t frame, time is represented by concentric spheres of different radii with the origin of the spacialaxes as their center. Although the space coordinates are bi-directional, time cannot be given a negative value thus the time coordinate only has one outgoing direction in this 3-d $s$-t frame. The advent of each new coordinate system (Cartesian, Galilean, etc) has ushered in new developments in physics and in mathematics. It might be worth while considering the advantages of a3-d s-t frame and seeing what potential developments it can offer [3] [4].

The line separating modern physics and classical physics is Special Relativity. In classical physics, space and time are absolute and independent. For example in classical physics, if a table is 3 meters in length, it is an absolute 3 meters, and 


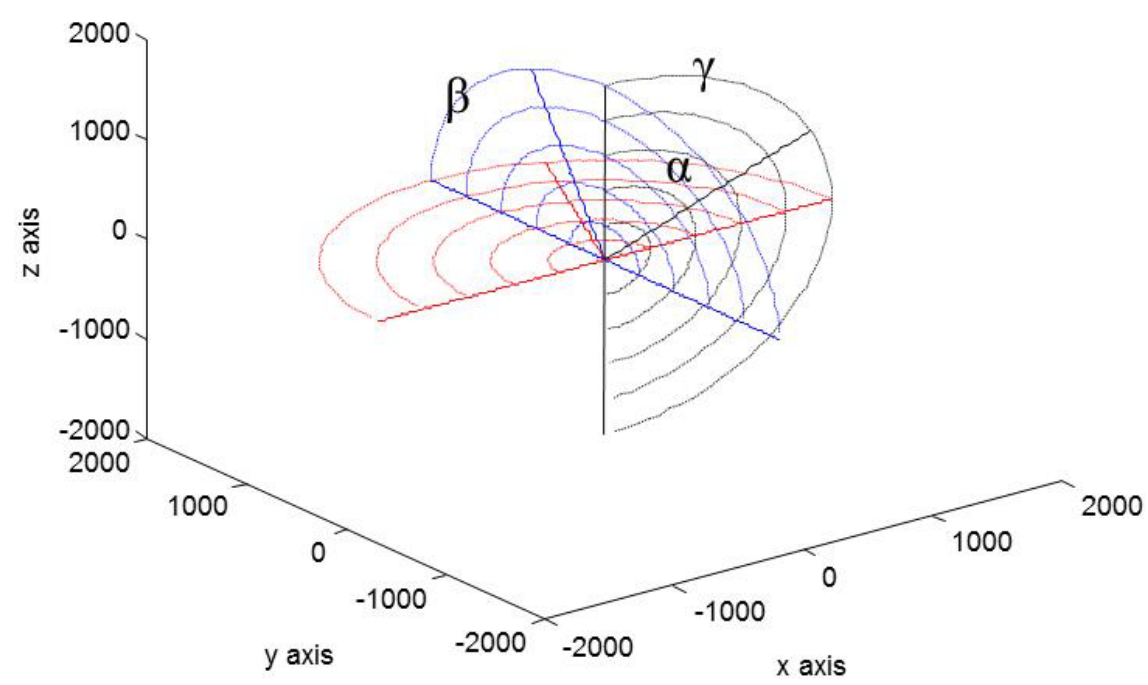

Figure 1. The construction of a 3-d s-t frame. The point with the property, $h(x(t))=\sqrt{r^{2}(t)-x^{2}(t)}$ on the $x-y$ plane can represent the location of the particle moving along the $x$-axis at time $t$. The point with the property, $h(y(t))=\sqrt{r^{2}(t)-y^{2}(t)}$ on the $y-z$ plane can represent the location of the particle moving along the $y$-axis at time $t$. The point with the properties, $h(z(t))=\sqrt{r^{2}(t)-z^{2}(t)}$ on the $z-x$ plane can represent the location of the particle moving along the $z$-axis at time $t$.

its length has nothing to do with where and when the table is measured. In classical physics, if an hourglass take 15 minutes to empty, it is an absolute $15 \mathrm{mi}$ nutes, and the time it takes for the sand to empty has nothing to do with where and when this emptying takes place. In modern physics, however, Einstein developed Special Relativity which showed that space and time are not absolute and are not independent, and measurements of length and time will not necessarily be the same for different observers. What is reality of length for the table? What is reality of time for the period of the event?

In Einstein's famous thought experiment: Einstein placed two photon guns in the middle of a car, one pointed towards the front wall and the other one pointing to the rear wall. The car moves with velocity $u$ relative to observers, who remain stationary on a platform. As the car passes observers on the platform both guns are fired at the same time. The speed of the photons is the speed limit for all particles in the universe, which is the speed of lightand measured to be $3 \times 10^{8} \mathrm{~m} / \mathrm{sec}$, a constant value for observers on any coordinate frame. Observers in the car will see the photons hit the front wall and rear wall, simultaneously. The observer on the platform will see the photon hit the rear wall of the car before the other photon hits the front wall of the car, because the photon pointed at the front wall will have a greater distance to travel.

Classical physics tells us, the simultineity of two events is absolute, so time and space are independent for observers on a pair of inertial frames [3] (platform and car) with relative velocity $u$. Einstein demonstrated that the simultineity of two events is relative, so time and space are dependent for observers on a pair of inertial frames with relative velocity $u$. Under the condition of the con- 
stant speed of light measured from observers on any frame, the simultineity of two events is dependent on observers' frames of reference. What is reality of two simultaneous events?

In a slightly modified version of Einstein's thought experiment, the photon is shot from the floor of the car on a moving train, directly vertical towards the ceiling (see in Figure 2). The frame of observers on the platform is $S$, the frame of observers on the train is $S^{\prime}$, and the train's speed is $u$ in the direction towards the right. Observer on the train: The photon flies vertically from the floor to the ceiling, where $h$ represents the path of the photon relative to those observers. Observers on the platform: The photon flies at a slant from the floor to ceiling, where $r$ represents the track of photon relative to those observers.

From the time the photon leaves the gun on the floor until the time it reaches the ceiling, the distance traveled by the train is $X$, as measured by observers on the platform. $r, X$, and $h$ form three sides of a right triangle, utilizing the Pythagorean theorem:

$$
h^{2}=r^{2}-x^{2}
$$

Substituting $c t^{2}$ for $h, c t$ for $r$, and $u t$ for $x$ and taking out the common factor $t$ squared on the right side of the equation, yields the following: $\left(c t^{\prime}\right)^{2}=\left(c^{2}-u^{2}\right) t^{2}$. Dividing both sides of the equation by $c$ squared, then taking the squared root on both sides, yields the time contraction formula:

$$
t^{\prime}=\left(\sqrt{1-\left(\frac{u}{c}\right)^{2}}\right) t
$$

because $u$ is always less than $c$, the factor $\sqrt{1-\left(\frac{u}{c}\right)^{2}}$ before $t$ must be less than 1 , which means that the time measured by observers on the train $t$ must less than the time measured by observers on the platform [5] [6]. As an example, let us assume the speed of the train as measured from an observer the platform is $0.6 c$, and the train travels for $25 \mathrm{sec}$ before the photon hits the ceiling. By in putting this data into the time contraction formula, we get $20 \mathrm{sec}$, which is the time it takes for the photon to hit the ceiling, as measured by observers on the train, which is less than the time $25 \mathrm{sec}$ measured from the platform.

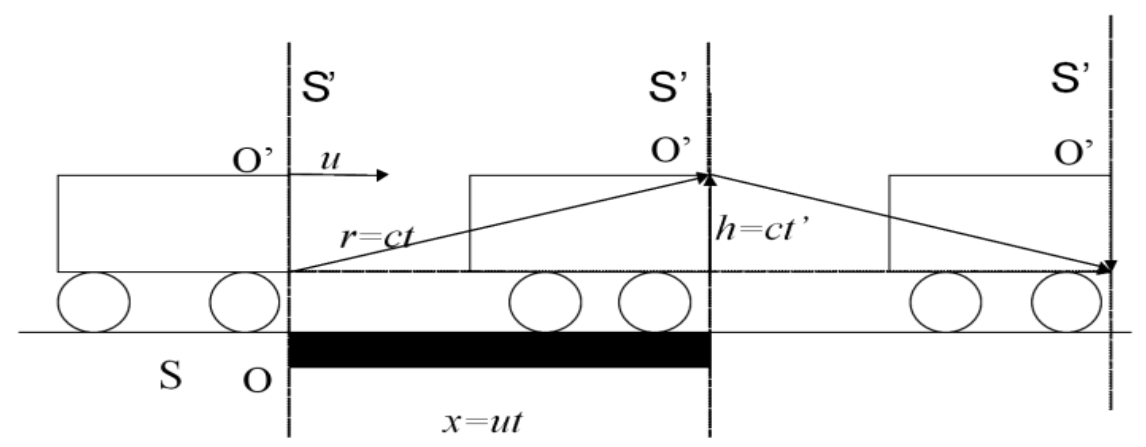

Figure 2. Modify Einstein's thought experiment by shooting a photon from the floor of a car of the moving train to the ceiling. The sides $r, x, h$ of a triangle form the right triangle. 
We continue our discussion of the above thought experiment, by multiplying both sides of the time contraction formula by $u$, which yields the following equation:

$$
u t^{\prime}=\left(\sqrt{1-\left(\frac{u}{c}\right)^{2}}\right) u t
$$

The distance measured by observers on the train is $u t^{\prime}$ and the distance measured by observers on the platform is $u t$, as shown in Figure 3. Making the appropriate substitutions to the previous formula, we derive the length contraction formula [7]:

$$
x^{\prime}=\left(\sqrt{1-\left(\frac{u}{c}\right)^{2}}\right) x .
$$

And using the previous example, where the speed of the train is $0.6 c$ and the train travel time of $25 \mathrm{sec}$ (relative to the platform), the train travels a distance of $u t=0.6 c \times 25 \mathrm{sec}=15 \mathrm{lsec}$, as measured by observers on the platform. By inputting this data into length contraction formula, we get the distance $12 \mathrm{lsec}$, which is the distance the photon travels as measured by observers on the train, which is shorter than the distance $15 \mathrm{lsec}$, as measured by observers on the platform.

One interesting example to consider: if the speed the train is equal to the speed of light. No matter how much time, $t$, has passed or how much distance, $x$, the train has traveled relative to an observer on the platform, time for observers on the train

$$
t^{\prime}=\left(\sqrt{1-\left(\frac{c}{c}\right)^{2}}\right) t=\left(\sqrt{1-(1)^{2}}\right) t=0
$$

is measured to be zero and distance for observers on the train

$$
x^{\prime}=\left(\sqrt{1-\left(\frac{u}{c}\right)^{2}}\right) x=\left(\sqrt{1-(1)^{2}}\right) x=0
$$

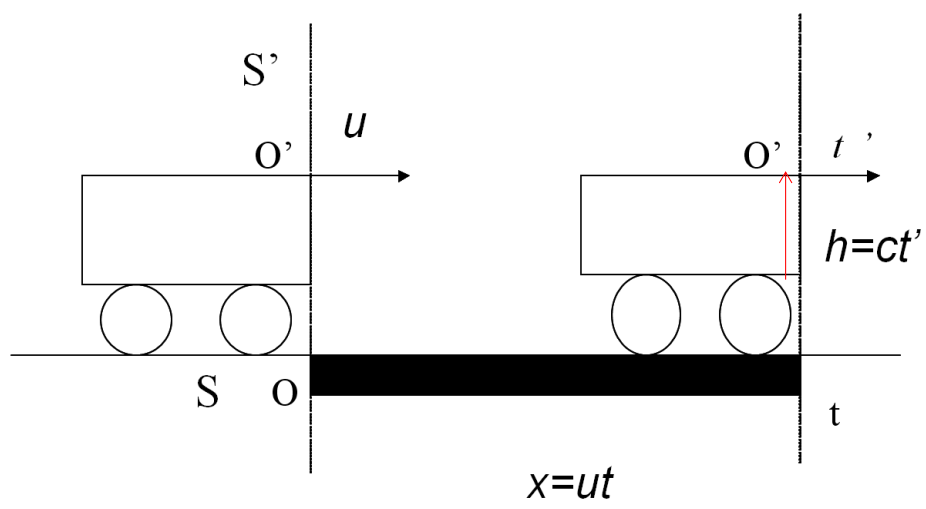

Figure 3. Modify Einstein's thought experiment by shooting a photon from the floor of a car of the moving train to the ceiling. The distance of the train traveling measured from observers on the platform is $x=u t$. The distance of the train traveling measured from observers on the train is $x^{\prime}=u t^{\prime}$. 
is measured to be zero. It shows that if we are able travel on the train (UFO) moving with the velocity of light, $c$, then we are able to reach anyplace with zero time and zero distance [1].

\section{Advantages in Applications of 3-d s-t Frames}

\subsection{The Shell Model of the Universe from Multiple Big Bangs Based on 3-d s-t Frame}

The Current Standard Model of the Universe based on the 4-d s-t frame asserts that the universe was generated from a single Big Bang event followed by inflation. There is no center to this universe, hence, no preferential reference frame to describe the motions of celestial objects. We propose a new Shell Model of the Universe based on the $3-\mathrm{d} s$-t frame, which contends that the universe was created from multiple, concentric big bangs. Accordingly, that origin presents itself as a unique, preferential reference frame, which furnishes the simplest description of the motions of galaxies in the cosmos. This is similar in manner to how planetary motion is more straightforwardly described via a sun-centered Solar System rather than an earth-centered one [8]. All galaxies residing on the same shell are at a corresponding age. The contention is that our universe is a conglomerate of numerous, unequally-matured shells created from multiple big bangs with a common origin and not simply a center-less collection of masses generated by a single Big Bang, per the Current Standard Model of the Universe. The appeal of the Shell Model of the Universe lies in its simplistic ability to resolve the paradox of quasars, explain the variability in Hubble's Constant, and solve the problematic accelerated expansion of the universe.

This Shell Model based on the 3-d s-t frame has been used to analyze the quasar paradox, the variability in Hubble's Constant, the accelerated expansion of the universe (attributed to dark energy by some), and stars located at the edge of a spiral galaxy moving with unexpected high velocity (attributed to dark matter by some) [8].

The Shell Model asserts that Hubble's “constant" varies because galaxies may reside on different shells and that redshift is not the right method to calculate distance [8]. A quasar has a rather large redshift, because the quasar and our galaxy are moving in opposite (not just different) directions [8]. It has nothing to do with distance. Stars located at edge of a spiral galaxy moving with unexpected high velocity with respect to our galaxy. If we are able to recalculate the velocity of stars located at edge of a spiral galaxy with respect to the center of multiple big bangs, the value should be much smaller [8]. There is no need of dark matter. The Shell Model postulates that there have been multiple big bangs and there will continue to be more. When there are enough big bangs, the collective mass of all galaxies will large enough to cause the universe to contract, which means that a contraction of the universe does not need to be explained using dark mass [8]. Each big bang produces a shell of galaxies having the same outward velocity. Galaxies on the shell related the more recent big bang should slow down the ve- 
locity of galaxies by gravitational pull on the outer shells related to older big bangs. As a result of the location and direction of travel of a galaxy, the relative velocity of this galaxy to our galaxy might be measured to be very large value [8]. If we are able to recalculate the velocity of galaxies with respect to the center of multiple big bangs, the value should be equal to expansion velocity of shells [8].Although galaxies have large redshifts with respect to our galaxy, they have nothing to do with accelerated expansion of the universe. There is no need of dark energy in the Shell Model of the Universe based on the 3-d s-t frame.

The origin of multiple big bangs presents itself as a unique, preferential reference frame, which furnishes the simplest description of the motions of galaxies in the cosmos. This is similar in manner to how planetary motion is more straight forwardly described via a sun-centered Solar System rather than an earth-centered one. All hidden factors behind strangemotions of galaxies, which cannot be explained in the Current Standard Model of the Universe based on the 4-d s-t frame, will disappear in the Shell Model of the Universe based on the 3-d $\mathrm{s}-\mathrm{t}$ frame.

\subsection{Quantum Mechanics}

When the motion of a micro quantum is described by emitting photons without being able to see the point of contact, the probabilities of two uncertain measurements (due to the rotation of a quantum and the wavelength a photon wave) are unavoidable. The Heisenberg uncertainty relation is proved by simply multiplying two wavelengths; the wavelength of a quantum matter wave and the wavelength of a photon wave [9]. This proof leads to the conclusion that the wavelength of a quantum matter wave, which is equal to $h / p$ postulated by de Broglie, is actually a probability wavelength, not a conventional wave length. Heisenberg uncertainty relation can be derived by multiplying a probability wavelength due to the rotation of a particle and the wavelength of a photon wave due to measuring medium [9]. This new approach may offer another view to look at the reality of M. Born's statistical interpretation about the wave function of the solution of Schrodinger's equation.

No matter how far away two particles are separated. If there is an interaction between these two particles by the medium (train) traveling with the velocity of light, then the medium affect two particles instantly. If the speed the medium is equal to the speed of light, no matter how much time, $t$, has passed or how much distance, $x$, the medium has traveled relative to an observer on the platform, time, $t^{\prime}$, for observers on the train is measured to be zero from Equation (5) and distance for observers on the train $x^{\prime}$, is measured to be zero from Equation (6). Although observers on the platform (two particles) find the affection taking time, but for observers on the medium (train) find affection instantly [1]. The reality of space and time discussed in Special Relativity should help us understand quantum entanglement without assuming the medium with infinite velocity. 


\subsection{Length, Time, Mass Are Not Fundamental Quantities}

All physical quantities are based on three fundamental concepts: distance, time, and mass. The three fundamental quantities are measured in terms of the three basic units of measure, which in the International System of units are: meter, second, and kilogram. The International System, or the SI system, is often referred to as the metric system. Human interactions, in industry and commerce among others, depend on measuring devices based on these units adopted in 1946 [10].

In the National Physics Laboratory in Great Britain, the definitions of the three fundamental standard units are: In 1975, the speed of light, $\mathcal{C}$, was known to be the fixed numerical value $299,792,458 \mathrm{~m} / \mathrm{sec}$. The meter is the length of the path traveled by light in a vacuum during a time interval of 1/299,792,458 of a second [10], because of $(299,792,458 \mathrm{~m} / \mathrm{sec})(1 / 299,792,458 \mathrm{sec})=1 \mathrm{~m}$. The second is the duration of $9,192,631,770$ periods of the radiation corresponding to the transition between the two hyperfine levels of the ground state of the cesium-133 atom [10]. The kilogram is the unit of mass; it is equal to the mass of the international prototype of the kilogram [10].

We suggest that the second is redefined in International System of Units (SI) as the time period of the event of the light travelled 299,792,458 $\mathrm{m}$ in vacuum, because of

$$
(299,792,458 \mathrm{~m}) /(299,792,458 \mathrm{~m} / \mathrm{sec})=1 \mathrm{sec} .
$$

Because of $c=299,792,458 \mathrm{~m} / \mathrm{sec}=\frac{\lambda_{1}}{T_{1}}=\frac{\lambda_{2}}{T_{2}}=\cdots=\frac{\lambda_{n}}{T_{n}}$, then we are able to define $\lambda_{1}, T_{1}$ as wavelength and period for red light, $\lambda_{2}, T_{2}$ as wavelength and period for orange light, $\ldots, \lambda_{n}, T_{n}$ as wavelength and period for purple light. The second in International System of Units is defined as the duration of 9,192, 631,770 periods of the radiation corresponding to the transition between the two hyperfine levels of the ground state of the cesium-133 atom. From the period $T$ equal to $T=\frac{1}{f}=\frac{1}{9,192,631,770 / \mathrm{sec}}=1.087827757 \times 10^{-10} \mathrm{sec}$ and $c=299,792,458 \mathrm{~m} / \mathrm{sec}=\frac{\lambda}{T}$, then the wavelength $\lambda$ should be equal to $0.032612255 \mathrm{~m}$ in order to make the ratio of $\lambda$ to $T$ equal to the velocity of light, $c$. Actually, both definition of the second and the meter in International System of Units are operational definitions using the fixed value of the velocity of light.

The kilogram in International System of Units is defined as the unit of mass; it is equal to the mass of the international prototype of the kilogram. Actually, it also is operational definition by equally gravitational force on the object and the international prototype from the balance of the scale.

It is a misconception to believe time and space possessing innate essentials. The reality is that without movement, we would not be able to measure the time elapsed or the distance traveled. If we want to discuss time and space, we cannot mention them separately and must not forget to acknowledge the events/phe- 
nomena/context that produced these attributes. We believe that velocity is much more fundamental than time and length, which are defined by velocity. Space and time are operational definitions by choosing velocity of light as fundamental quantity.

We can also show that force is much more fundamental than mass, which is defined by gravitational force or inertial force [10]. Mass is operational definitions by choosing force as fundamental quantity. This leads us to conclude that events are much more fundamental/basic than the attributes (time, length, and mass) that objects possess, which are derived/defined by events.

\section{Conclusion}

Three-dimensional space-time frames (3-d s-t frames)are better suited to describing the motion of a particle in Special Relativity than Galilean frames and Minkowski's frames. 3-d s-t frames provide an alternate framework for interpreting astronomical phenomena and quantum mechanics and may yield other potentially meaningful insights when applied to other areas of study.

The construction of 3-d s-t frames is based on a photon with the velocity of light, $c$ and a pair of inertial frames with the relative velocity, $u$. From Figure 2, time is defined as $t^{\prime}=h / c$ and from Figure 3, distance is defined as $x^{\prime}=u t^{\prime}$ by observers on the train. From Figure 2, time is defined as $t=r / c$ and from Figure 3, distance is defined as $x=u t$ by observers on the platform. From this, we are able to derive the time contraction formula, $t^{\prime}=\left(\sqrt{1-\left(\frac{u}{c}\right)^{2}}\right) t$ and length contraction formula $x^{\prime}=\left(\sqrt{1-\left(\frac{u}{c}\right)^{2}}\right) x$. Because $t^{\prime}=\frac{h}{c}$ and $t=\frac{r}{c}$ from the Figure 2, and if $u=0$, then $x=0$ and $r=h$, therefore $t^{\prime}=t$. Of course the time measured by observers on the platform should be same as the time measured by observers on the train staying still with zero velocity.

The previous discussion helps us define operational definitions of distance and period of time traveled by the train from $c$ and $u$ as fundamental quantities. Using 3-d s-t frames, the reality of space and time is understood through a photon with the velocity of light, $c$, and a pair if inertial frames with relative velocity, $u$. In addition, mass is defined as operational definition by fundamental quantity force. It means that we are able to understand reality of mass through fundamental quantity force.

From this discussion concerning space, time, and mass, we induce the following statement of Contextual Reality [11]: We are able to cognize the being of an individual through the existence of phenomena and are able to describe the properties of that individual through the individual's possession of contextual attributes. We are not able to cognize the being of an individual through the existence of individual itself and are not able to describe the properties of that individual through the individual's possession of innate essentials. For example, we are able to label a particle to be an electron, because of the way it bends when 
a magnetic field is applied and not because the particle possessing certain innate essentials. The particle only possesses the contextual attributes of an electron, i.e. the way it bends, in the context of the event where a magnetic field is applied.

We should try to reconstruct theories of physics by searching for fundamental events (phenomena) instead of searching for fundamental objects. Objects do not possess innate essentials; the attributes are derived through the events by which the object is examined. Using this approach may lead to a better understanding of the reality of the universe.

The statement of Contextual Reality and also be applied to our awareness of others. For example, if we must label an individual as being "bad tempered", it is important to provide the particular context in which that individual displayed that attribute. While an individual may display a "bad temper" during frequent events/occurrences, that individual still does not possess that innate essentials absolutely. Remembering that a statement concerning an individual's qualities should always be accompanied with context helps turn our judgment into understanding.

\section{References}

[1] Chen, T. and Chen, Z. (2016) Special Relativity in Three-Dimensional Space-Time Frames. International Journal Astronomy and Astrophysics, 6, No. 5. http://dx.doi.org/10.4236/jmp.2016.6-4

[2] Chen, T. and Chen, Z. (2005) Motions of Particles Described in a Three-Dimensional Space-Time Frame. The Proceeding of the Tenth Asian Technology Conference in Mathematics, Kore, 15-20 December 2005. http://epatcm.any2any.us/EP/EP2005/2005P163/fullpaper.pdf

[3] Chen, T. and Chen, Z. (2008) A Pair of Inertial Frames versus an Inertial Frame. Concepts of Physics, 5, No.3. http://merlin.fic.uni.lodz.pl/concepts/www/V_3/523.pdf

[4] Chen, T. and Chen, Z. (2016) A Bridge Connecting Classical Physics and Modern Physics. Journal of Modern Physics, 7, 1378-1387 https://doi.org/10.4236/jmp.2016.711125

[5] Chen, T. and Chen, Z. (2012) The Advantage of Three-Dimensional Space-Time Frames. Frontiers in Sciences of Sciences Academic Publisher, 2, 18-23. https://doi.org/10.5923/j.fs.20120203.01

[6] Chen, T. and Chen, Z. (2008) The Paradox of Twins Described in a Three-Dimensional Space-Time Frame. The Proceeding of the Tenth Asian Technology Conference in Mathematics, Bangkok, Thailand, 15-19 December 2008. http://atcm.mathandtech.org/EP2008/papers_full/2412008_15023.pdf

[7] Chen, T. and Chen, Z. (2009) Time Dilation and Length Contraction Shown in Three-Dimensional Space-Time Frames. Concepts of Physics, 6, No. 2. http://merlin.phys.uni.lodz.pl/concepts/www/VI_2/221.pdf

[8] Chen, T. and Chen, Z. (2016) The Shell Model of the Universe: A Universe Generated from Multiple Big Bangs. Special Issue on Research on Gravitation, Astrophysics and Cosmology Journal of Modern Physics, 7, 611-626. https://doi.org/10.4236/jmp.2016.77062

[9] Chen, T. (2006) The Heisenberg Uncertainty Relation Derived by Multiplying Matter Wavelength and Light Wavelength. Concepts of Physics, 3. 
http://merlin.fic.uni.lodz.pl/concepts/2006_1/2006_1_1.pdf

[10] Chen, T. and Chen, Z. (2016) Time, Length, Mass Are Derived Quantities. Journal of Modern Physics, 7, No. 10. https://doi.org/10.4236/jmp.2016.710108

[11] Chen, T. Contextual Reality. Jern Cheng Press, Taiwan.

\section{Scientific Research Publishing}

Submit or recommend next manuscript to SCIRP and we will provide best service for you:

Accepting pre-submission inquiries through Email, Facebook, LinkedIn, Twitter, etc. A wide selection of journals (inclusive of 9 subjects, more than 200 journals)

Providing 24-hour high-quality service

User-friendly online submission system

Fair and swift peer-review system

Efficient typesetting and proofreading procedure

Display of the result of downloads and visits, as well as the number of cited articles

Maximum dissemination of your research work

Submit your manuscript at: http://papersubmission.scirp.org/

Or contact jamp@scirp.org 